\title{
Differential Signaling Compromises Video Information Security through AM and FM Leakage Emissions
}

\author{
Pieterjan De Meulemeester, Bart Scheers, and Guy A.E. Vandenbosch, Fellow, IEEE
}

\begin{abstract}
Video display units (VDUs) using differential signaling technology significantly increase the risk of compromising video information security through leakage emissions. This paper shows that differential signaling cables act as substantial video leakage sources. A concept is proposed which explains the video leakage principles of VDUs using differential signal cables such as the HDMI (high-definition multimedia interface) cable, DVI (digital visual interface) cable and the LVDS (low-voltage differential signaling) cable. The emanations of the LVDS cable are closely examined by measuring simultaneously the differential video signal on the LVDS lines and its near- and far-field leakage emissions. From these measurements several conclusions are drawn which give new insights into the video eavesdropping risk of VDUs using differential signaling methods. Furthermore, a novel video image reconstruction method is proposed which exploits the compromising emanations of a video display unit (VDU) by using frequency demodulation techniques. This paper shows that leaked video emanations of VDUs using differential signaling cables are not only amplitude modulated (AM) but also frequency modulated (FM). This strongly implies that the possible algorithmic toolset of malicious video eavesdroppers is much larger than currently assumed. This paper investigates several VDU setups at a distance of 10 meters, including an ultra-high-definition video display, three different HDMI cables and two notebooks. Additionally, the AM-based and FM-based video image reconstruction results are discussed and compared.
\end{abstract}

Index Terms-Side-channel attack, compromising emanations, TEMPEST, electromagnetic attack, video eavesdropping, video leakage, information security, computer security, differential signaling, HDMI, LVDS, frequency modulation (FM), amplitude modulation (AM).

\section{INTRODUCTION}

$\mathbf{I}$ NFORMATION processing systems make up an inherent part of our modern society. The level of information security is crucial for maintaining the integrity of these systems. In the last decades, the field of computer security has seen much progress due to the rapid growth of computer networks and smart devices. Increased cybersecurity measures have driven third parties who have an interest in data theft to look into other methods. Side-channel attacks, for instance, circumvent these cybersecurity measures by exploiting physical phenomena which result in the compromise of data security. This scope of this research work is narrowed down to the compromising emanations of video display units (VDUs) [1]. Each VDU inevitably leaks into the electromagnetic (EM) farfield due to poor shielding, unwanted common and differential modes traveling on wires, poor connectors, fortuitous emitters, etc... [2]. It is shown by several research works [3]-[8] that these leaked emanations compromise data security. This specific side-channel attack poses a risk for data security especially because it is able to obtain secured data without having any physical access to the target system. It is also impossible to detect if data are compromised due to the passive nature of the attack.

This data security threat has been investigated before by defense institutions who have in turn established different emission standards referred in literature as emission security (EMSEC) and TEMPEST [9]. In 1985, this threat was revealed by W. Van Eck to the scientific community for the first time. He succeeded in recovering the video image displayed on a cathode ray tube (CRT) display. However, decades later, this threat still isn't well-documented and understood by the research (scientific) community. Today's VDUs are still very much affected by this eavesdropping risk as shown in the following works [4]-[8], [10]-[15]. These confirmed that VDUs in general and video data signaling cables such as VGA (video graphics array), DVI (digital visual interface), HDMI (high-definition multimedia interface) and LVDS (low-voltage differential signaling) suffer from compromising emanations. Most of these leakage sources employ differential signaling techniques to transmit data at high speeds except for the VGA cable.

This research work examines the video leakage risk of differential signaling cables in more detail. Consequently, this paper proposes novel concepts based on near- and farfield measurements that explain the video leakage emissions originating from differential signaling cables. First, it looks into possible leakage sources at the signaling level by analyzing both the physical layer and the data link layer. Next, it examines its leakage response in the near- and far-field using various video setups and evaluates this response against previous findings. Subsequently, this research work is the first that looks more closely into the possibility of detecting emanated video signals with frequency demodulation techniques. Until now, it is generally accepted that leaking video emanations are amplitude modulated, and as far as the authors can tell, all research up to this moment has exploited that fact. The results in this paper clearly confirm that leaking video emissions not solely contain amplitude modulated signals but also frequency modulated signals. These results strongly imply that the possible algorithmic toolset of malicious eavesdroppers is much larger than currently assumed. By combining FM-based and AM-based video image reconstructions extra video image information can be obtained. Hence, the risk of a successful eavesdrop attack increases. 
Several video cases are examined e.g. setups that include a variety of HDMI cables which differ in length and manufacturer, two notebooks, an ultra-high-definition (UHD) LCD (liquid-crystal display) with a pixel resolution of 3840x2160 placed at a distance of 10 meter.

Section II discusses and proposes a concept which explains the video image leakage emanations originating from differential signaling cables based on various measurements. This section is divided as follows,

- Video Leakage Sources of Differential Signaling Cables (II-A).

- Video Leakage Measurement Analysis of an LVDS Cable (II-B).

- A Video Image Leakage Concept of VDUs using Differential Signaling (II-C).

- Modulation of Captured Video Leakages in the Far-Field (II-D).

- Video Leakage Measurement Analysis of a VDU Setup at a 10-Meter Distance (II-E).

- Video Image Leakage Detection, Synchronization Recovery and Reconstruction (II-F).

- The Tested VDU Setups (II-G).

Section III discusses the results of AM-based and FM-based video reconstruction of two different VDU test cases. Section IV concludes.

\section{Video IMAGE LEAKAGE EMANATIONS ORIGINATING FROM DIFFERENTIAL SIGNALING CABLES}

\section{A. Video Leakage Sources of Differential Signaling Cables}

Most modern VDUs employ differential signaling cables to transmit video information to the display. Popular differential signaling cables are DVI, HDMI and LVDS cables. The latter is an internal cable and the others external. These cables can act as video leakage sources due to the combination of high serial data throughput at high frequencies and shielding imperfections which results in the cable behaving as a wire antenna. Despite the fact that the differential signaling scheme is designed in such a way to improve EMC (electromagnetic compatibility) and immunity against EMI (electromagnetic

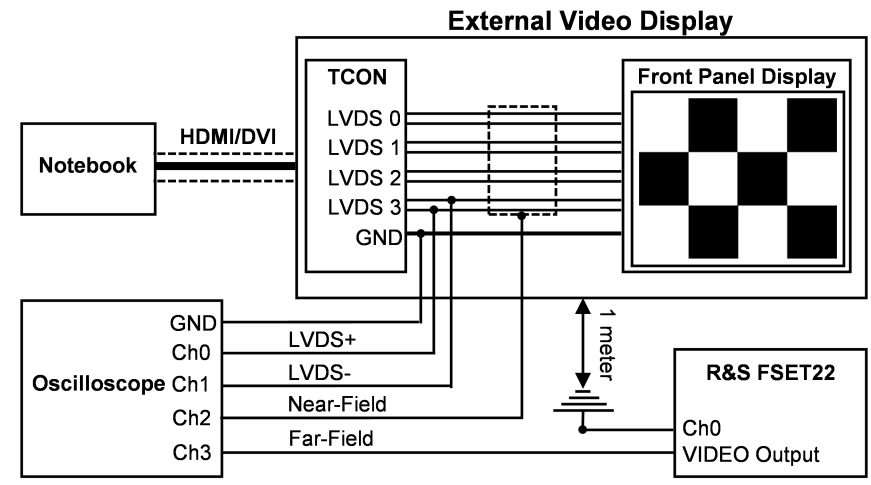

Fig. 1: Schematic of a VDU measurement setup that acquires the LVDS signal, the common-mode noise signal on the LVDS, the near-field using an H-field probe and the far-field using a log-periodic dipole antenna at a distance of one meter. interference). This is achieved by employing two complementary signal lines which are the exact opposite of each other and both symmetrically referenced to the ground. In addition, differential receivers are employed which cancel out the common-mode noise and increase the SNR (signal-to-noise ratio) of the signal. However, the differential signal itself can produce a significant common-mode noise signal when the two complementary signals are slightly distorted. In reality, a small delay on one of the lines can be introduced or the rise/fall time of the signal drivers can differ on both lines. This creates an asymmetrical situation on both lines which produces a common-mode noise signal. This imbalance on both lines also worsens significantly when the length of the cable increases [16], [17].

When the differential signal is distorted, common-mode noise pulses are generated at the rise and fall location of the original square wave signal. These pulses will be emitted into the EM near- and far-field. Therefore, these captured leakage signal will have a certain correlation with the original video signal. To understand what is captured, a differential signal employed by the LVDS cable is explored more in detail by measuring the video signal on the LVDS lines and its leakage emissions in the near- and far-field.

\section{B. Video Leakage Measurement Analysis of an LVDS Cable}

In Fig. 1, a schematic is depicted of the VDU measurement setup used to examine the video leakage relation between the near-field, the far-field, the differential signal and the common-mode noise signal on the LVDS lines. The test video image represents a checkerboard pattern. The results of these measurements are visualized in Fig. 2. The captured time period equals $18 \mu \mathrm{s}$ and the signal is sampled at $25 \mathrm{GS} / \mathrm{s}$. This time period roughly corresponds to one video line. The LVDS measurement is realized using a differential probe that determines the differential voltage of the LVDS+ and LVDSsignal line. This is realized by uncovering the LVDS cables of a VDU which were wired using twisted pairs. Subsequently, the H-field of all the LVDS cables is measured as depicted in Fig. 1. These LVDS cables consist of the measured LVDS line and the other three which hold the other color and

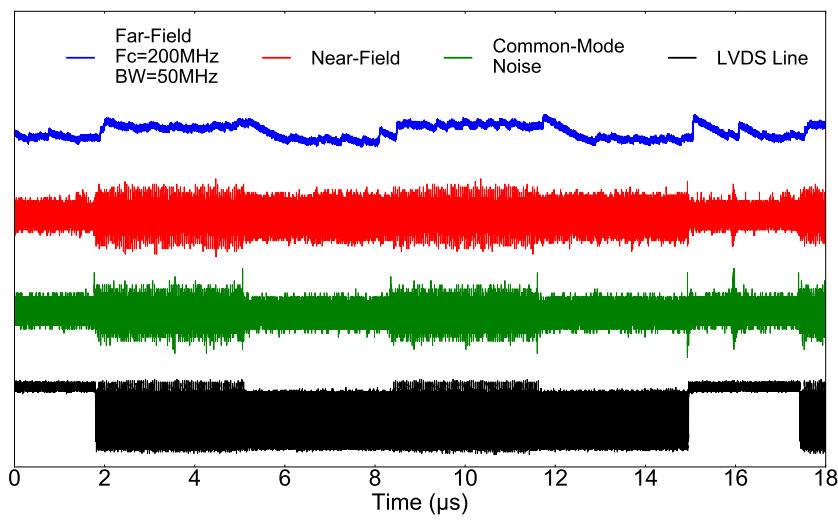

Fig. 2: Results of the measurement setup shown in Fig. 1. The signals are sampled at $25 \mathrm{GS} / \mathrm{s}$. The far-field is captured at the center frequency of 200 $\mathrm{MHz}$ with a bandwidth of $50 \mathrm{MHz}$ and is amplitude demodulated. 
synchronization information. The signaled bit codes on the other LVDS lines are identical due to the used test colors white and black. Finally, the far-field is captured from a 1meter distance using a log-periodic dipole antenna (LPDA) and a receiver. These 4 signals are sent to an oscilloscope and sampled at $25 \mathrm{GS} / \mathrm{s}$.

According to Fig. 2, an increase in bit transitions results in an increase of common-mode noise. This is in agreement with the findings of previous works [16], [17]. This commonmode signal is caused by possible imbalances on the line drivers. Parasitic and charging effects on the line drivers are the most probable causes for these imbalances. This results in a different rise/fall time of the square wave or introduces a small delay on one of the lines. The common-mode noise signal leaks into the near-field as shown in Fig. 2 which reveals a distinct correlation between the near-field and commonmode noise signal. Hence, the common-mode noise contributes significantly to the video leakage emissions.

For the far-field measurement displayed in Fig. 2, the antenna is connected to a superheterodyne receiver (Rohde \& Schwarz FSET22) which is tuned to a center frequency of $200 \mathrm{MHz}$ with a bandwidth of $50 \mathrm{MHz}$. The captured far-field leakage signal is amplitude demodulated, hence it returns the envelope of the leakage pulses. In addition, due to the lower bandwidth of $50 \mathrm{MHz}$ compared to the 325 $\mathrm{MHz}$ of the differential video signal, the bandwidth reduction results in a signal integration over a specific time interval (hence the strength of the captured signal peaks relates to the number of high frequency pulses during the time frame of 20 nanoseconds). The far-field results show a clear correlation with the common-mode noise signal and the near-field.

It should be taken into account that the leakage effects for this measurement are increased due to the probing of the LVDS cable. The main goal was to examine both the video differential signal running through the LVDS cable and its leakage response simultaneously.

\section{A Video Image Leakage Concept of VDUs using Differen- tial Signaling}

The proposed video image leakage concept is realized by analyzing both the physical layer and data link layer used by the video signaling methods. The physical layer, in this case, is the differential signaling lines and the data link layer corresponds to the video signal encoding schemes. The next part of this paper describes the proposed concept by relying on the measurements results of the previous section.

TABLE I: Tested VDU Setups at a measurement distance of 10 meters.

\begin{tabular}{|c|c|c|}
\hline $\begin{array}{l}\text { Test } \\
\text { Setup }\end{array}$ & Notebook & HDMI Cable \\
\hline I.A & Notebook I & $\begin{array}{l}\text { HDMI v1.4 cable A } \\
\text { - cable length of } 1.5 \text { meters }\end{array}$ \\
\hline II.A & Notebook II & $\begin{array}{l}\text { - HDMI v1.4 cable A } \\
\text { - Cable length of } 1.5 \text { meters }\end{array}$ \\
\hline II.B & Notebook II & $\begin{array}{l}\text { - HDMI v1.4 cable B } \\
\text { - Cable length of } 3 \text { meters }\end{array}$ \\
\hline II.C & Notebook II & $\begin{array}{l}\text { - HDMI v1.4 cable C } \\
\text { - Cable length of } 1.5 \text { meters }\end{array}$ \\
\hline
\end{tabular}

First, it should be mentioned that LVDS is, in principle, only a physical layer specification hence it does not specify the encoding of the transmitted signal. However, over the years, it has become a synonym for the front panel display link (FPDlink) which is located between the timing controller (TCON) and the front panel display as shown in Fig. 1. The FPD-link does use a certain encoding scheme standardized by VESA (Video Electronics Standards Association). In this paper, the term LVDS cable is also used as a synonym for the FPD-link.

The pixel's information displayed on a display is represented by 8 bits which define its color and brightness. The specific bit code will determine the number of bit transitions which, in turn, will affect the common-mode noise signal on the LVDS line as explained before. The bandwidth needed to capture each bit separately in the EM near- or far-field is in the order of $325 \mathrm{MHz}$ due to the high data rate of the LVDS cable which is in the order of $650 \mathrm{Mbit} / \mathrm{s}$ or higher. Capturing such a large bandwidth of the EM far-field will result in the intake of a large amount of noise making the video leakage signal very hard to detect. It is, however, not necessary to capture each bit individually to reconstruct the original video image. The pixel information is described by a bit word hence by detecting a change in bit words, a difference in the pixel value can be detected. This difference defines a contrast in the reconstructed video image. Each bit word contains multiple 0/1 or $1 / 0$ bit transitions which produce a common-mode noise signal as seen in Fig. 2. By integrating the leakage pattern originating from one bit word, a specific leakage strength is captured. However, it should be noted that not every bit word has a unique leakage response because some bit words have the same number of bit transitions.

The LVDS coding scheme maps 28 bits on 4 LVDS lines during one clock cycle if a color depth of 8 bits is used otherwise it maps 18 bits on three LVDS lines if a color depth of 6 bits is used. Hence 24 or 18 bit slots are allocated for the color information and 3 bit slots for synchronization information and a control bit. This implies that only 7 bits are signaled on one LVDS line and not 8 [18]. An HDMI or DVI cable employs the TMDS (transition-minimized differential signaling) technology, which is based on an 8 bit to 10 bit encoding scheme. This encoding scheme should improve the DC balance across the line, however this is not always the case [4]. Due to multiple differential lines, the global DC balance is not always preserved [19]. The HDMI and DVI use 4 TMDS lines of which three lines are dedicated to the RGB colors and one line for the synchronization/clock information.

The main differences between the TMDS signaling and

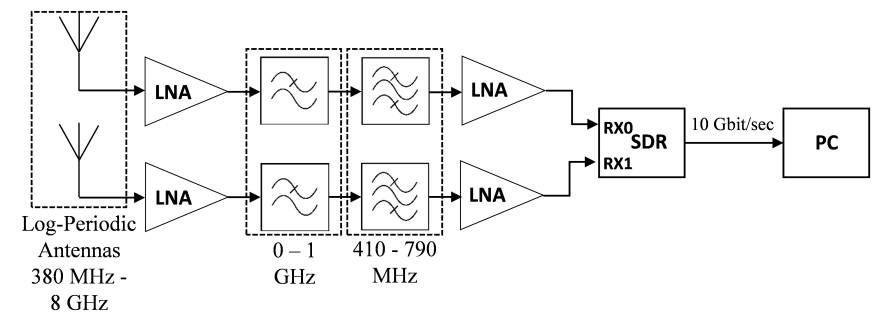

Fig. 3: Signal-acquisition and processing chain. 
LVDS signaling besides the $8 \mathrm{~b} / 10 \mathrm{~b}$ encoding is that color information on the LVDS lines is assigned across 4 lines or 3 lines depending on the color depth which is not the case for the HDMI and DVI cable which always assigns one color (10 bits) per line. Also, the HDMI cable supports multiple signal lines that do not contain video data such as a hot plug line, a $+5 \mathrm{~V}$ supply line, an utility line, a sound data line, etc... These signal lines will only add noise to detected video leakage emissions. Further details of the employed encoding schemes are not covered, because they are beyond the scope of this paper. In essence, the video encoding schemes transmit the video information in a sequential fashion which results in a correlation in time domain between the video leakage information and the original video signal.

Overall, the mentioned differences of the various signaling methods will affect the leakage response of every cable to some degree and will therefore result in distinct video leakage characteristics. Hence this distinct video leakage is defined by the physical layer and data link layer. The differential signaling principle which acts as the physical layer enables the video leakages and characterizes the video leakage frequency spectrum. In turn, the video encoding scheme which acts as the data link layer defines the captured video leakage information by how many bit transitions are signaled in sequence. In other words, both the physical and data link layer affect the common-mode noise signal which in turn determines the captured video leakage signal.

The next measurements examines the leakage of two VDU setups which are described in Table $\mathrm{I}$ as $I . A$ and $I I$.A. For these test setups, the VDU is untouched; hence it represents a typical office VDU which could be targeted. The results of these measurements enable further assessment and evaluation of the proposed video image leakage concept. Before these results are discussed, a brief overview is given of the modulation of the video leakage signal.

\section{Modulation of Captured Video Leakages in the Far-Field}

The nature of the video image leakage emanations is generally accepted by the research community to be amplitude modulated [3], [4], [7], [8], [11], [12], [20]. In other words, the information contained in the leakage channels reveals the change in amplitude of the original video data stream. The general concept is that the employed square wave signaling of
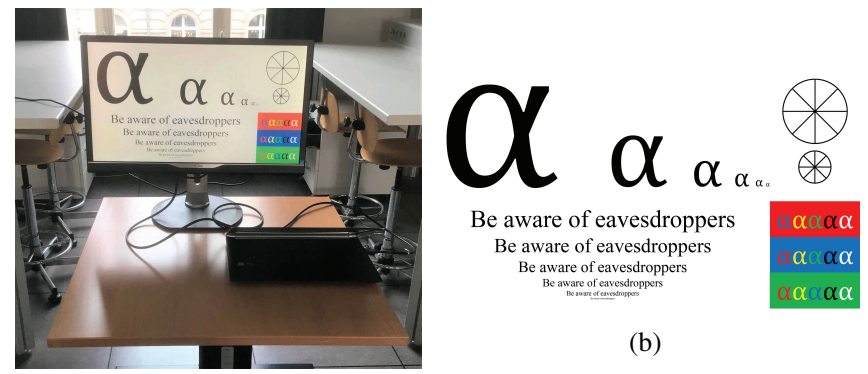

Be aw Be aware of eavesdroppers Be aware of eavesdroppers
Be aware of eavestroppers

(b)

(a)

Fig. 4: (a) Test setup II.A. (b) Test video image. The test video image contains Time New Roman font sizes of 5, 15, 25, 35, 45 and 55 points and $\alpha$ letters with fonts sizes of 15, 30, 60, 120, 240 and 480 points.

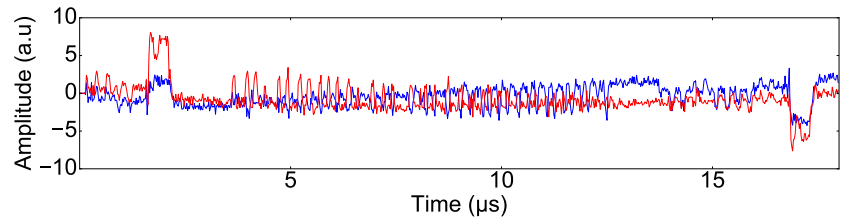

(a)

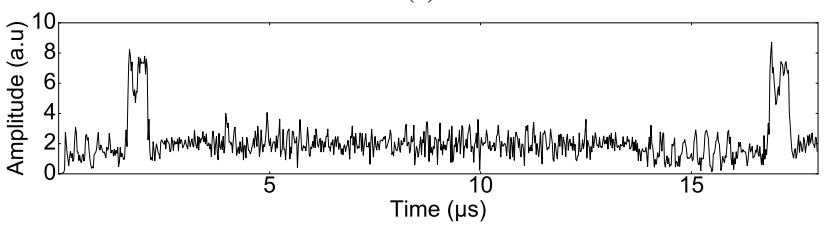

(b)

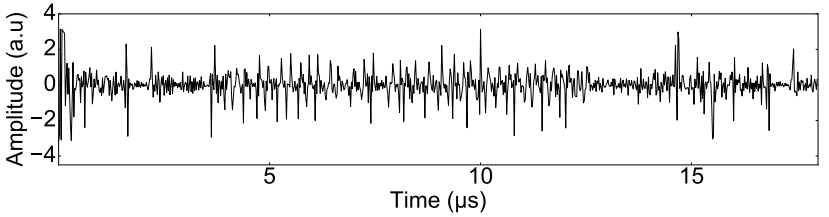

(c)

Fig. 5: Capture of the video leakage emanations of test setup II.A at a $f_{\text {center }}$ of $525 \mathrm{MHz}$ with a bandwidth of $50 \mathrm{MHz}$. The captured time period equals one video line. (a) IQ signal (blue line / red line resp.). (b) Amplitude demodulated signal. (c) Frequency demodulated signal.

the clock signal contains many harmonics which are emitted in the far-field due to the rapid change in current. These harmonics contain high frequencies which will act as carrier waves on which transmitted and processed data is induced [4], [21]. The content of the leaked emanations is strongly determined by how the video information is processed and transmitted [22]-[24]. In the case of parallel data paths that transmit the synchronization and the color value information of each pixel, the leakage emanations will hold a distorted amplitude modulated signal of superposed color and synchronization video information. Due to the superposition, the exact information of pixel color and synchronization is lost. Several research works have proposed a video image reconstruction method that successfully recovers the synchronization information of the VDU and the pixel's color information in terms of grayscale values solely from the video leakage emanations [6]-[8].

However the proposed video image reconstruction methods are based only on the AM of the leaking video emanations. The research work [25] confirmed that leaking emanations of IC (integrated chips), I/O connectors and data signaling paths are frequency demodulated and not only amplitude demodulated. However, their work did not cover video signal leakages.

This research work shows that video leakage emanations are also frequency modulated. Consequently, a novel video reconstruction method based on frequency demodulation is proposed. The knowledge that the leaked emanations hold distorted amplitude and frequency modulated signals can be exploited to increase the success of synchronization retrieval and video image reconstruction.

In our previous studies [8], [26], the captured data are amplitude demodulated using an asynchronous complex squarelaw envelope detection technique. In this work, beside an 


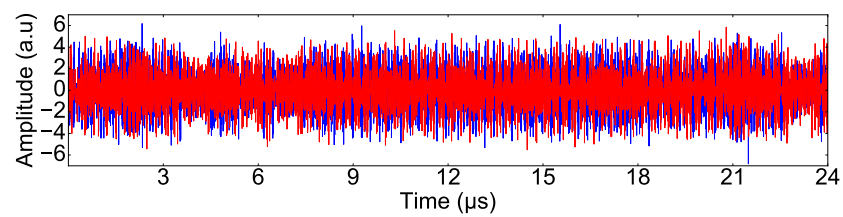

(a)

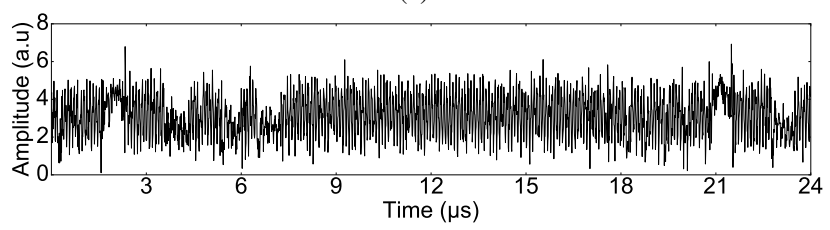

(b)

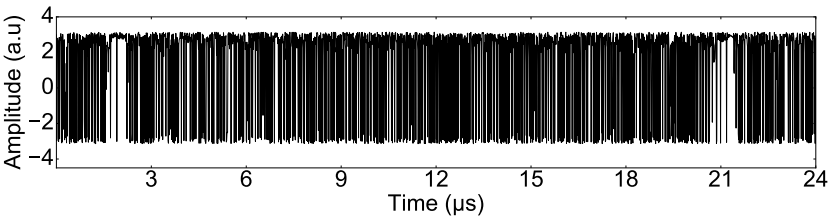

(c)

Fig. 6: Capture of the video leakage emanations of test setup I.A at the $f_{\text {center }}$ of $580 \mathrm{MHz}$ with a bandwidth of $100 \mathrm{MHz}$. The captured time period equals one video line. (a) IQ signal (blue line / red line resp.). (b) Amplitude demodulated signal. (c) Frequency demodulated signal.

amplitude demodulation, a frequency demodulation method is applied which is based on the following equations

$$
\begin{gathered}
x[n]=A e^{j 2 \pi\left(f T_{s} n+\phi_{0}\right)}, \\
\omega[n]=2 \pi f[n]=\frac{\Delta \phi[n]}{T_{s}}=\frac{1}{T_{s}} \arg \left(x[n] \cdot x^{*}[n-1]\right) .
\end{gathered}
$$

The equations are formulated with respect to a discrete time domain in which $f[n]$ represents the instantaneous frequency at sample $n, x[n]$ represents the complex IQ value of sample $n$, * the complex conjugate, $T_{s}$ the sampling period, $A$ the amplitude and $\phi$ the phase. This specific frequency demodulation method results in the instantaneous frequency and is invariant to the amplitude of the incoming signal. This is in contrast with some other frequency demodulation methods which are affected by signals containing AM and are dependent on the incoming amplitude such as a Foster-Seely discriminator [27]. Due to the fact that the leakage signals hold AM and FM information simultaneously, applied demodulation methods need to have a specific directivity towards a certain modulation to understand the nature of the leaking emanations.

\section{E. Video Leakage Measurement Analysis of a VDU Setup at a 10-Meter Distance}

To capture the leaked emanations, a signal-processing chain is used which consists out of two log-periodic dipole array antennas (LPDAs), LNAs (low-noise amplifiers), band-pass filters and a software-defined radio (SDR) as seen in Fig. 3. The LPDAs act as a phased array. The SDR employs a nearzero IF receiver which has a frequency range from DC (direct current) to $6 \mathrm{GHz}$. It IQ modulates the captured signal at a maximum sample rate of 200 Msps with a 14 bit resolution. The SDR feeds the sampled signal to a PC (personal computer) using a $10 \mathrm{Gbit} / \mathrm{s}$ Ethernet cable. The test setup II.A is visualized in Fig. 4a with the test video image in Fig. 4b.

The video leakage emanations of the test setup II.A are captured and analyzed. The $20 \mu \mathrm{s}$ captured IQ signal is shown in Fig. 5a and its demodulation in amplitude and frequency in Fig. 5b and Fig. 5c respectively. The IQ signal contains multiple pulses which show characteristics identical to the previously discussed leakage responses in section II-B. Hence these pulses are correlated to bit word transitions signaled through a differential cable. For this setup the main leaking differential cable is the HDMI cable as determined in section III-B when multiple video setups are tested of which only the HDMI cable is changed between the setups. The most interesting observation of the results in Fig. 5 is that the captured pulse strengths, rise and fall times of the pulses and the width of the pulses observed in the IQ signal leak video information. Hence this information is obtained by employing amplitude and frequency demodulation respectively, this is clearly visualized in Fig. 5b and Fig. 5c. The frequency demodulation captures the beginnings and endings of the pulses in the IQ signal and the rise and fall time of the IQ pulses correspond to the amplitude of the frequency demodulated signal. The amplitude demodulation captures the pulse strengths but also the width of the pulses.

In Fig. 6, the same measurements are done for the test setup of $I . A$. These measurements result in a less clear relation between the captured leakage signal and the video differential signal. The captured signal seems to be much noisier than the captures of test setup of $I I$.A. However, this noise is actually a high frequency oscillation that is superposed on the video leakage. This oscillation might be caused by coupling effect inside the HDMI cable due to poor shielding. This superposed oscillation does not affect the SNR of the reconstructed video image which is revealed in section III-A.

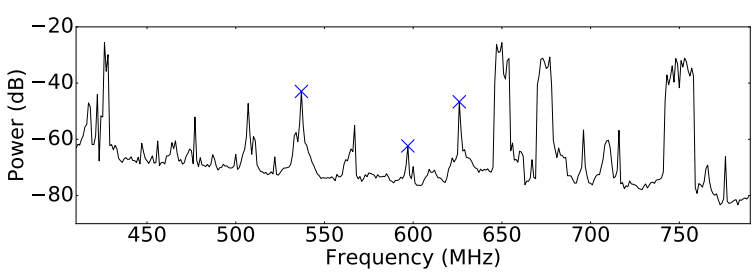

(a)

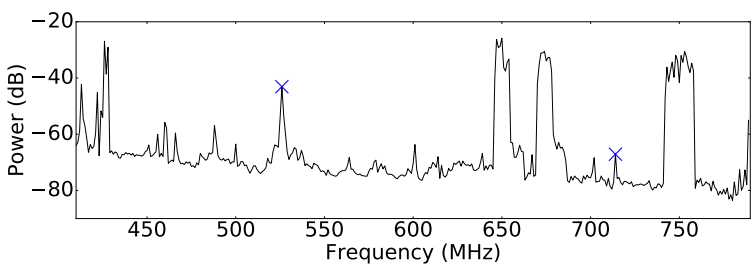

(b)

Fig. 7: Frequency spectra of test setups $I . A$ and II.A from $410 \mathrm{MHz}$ to 790 $\mathrm{MHz}$ with a resolution of $1 \mathrm{MHz}$ including the detected leakage channels indicated by a blue cross. Both spectra are averaged in time. (a) Test setup I.A. (b) Test setup II.A. 


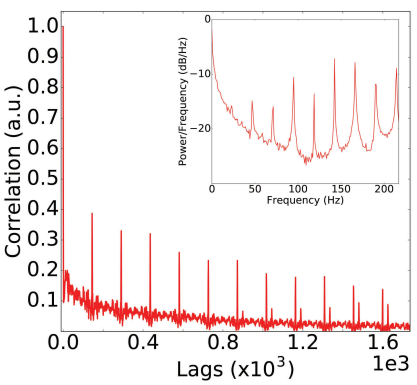

(a)

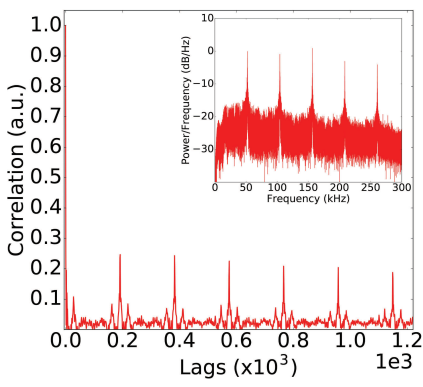

(c)

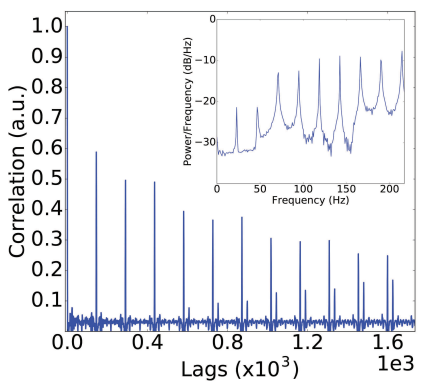

(b)

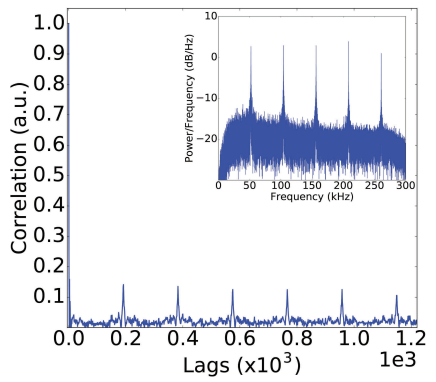

(d)

Fig. 8: Synchronization plots of test setup I.A at a $f_{\text {center }}$ of $580 \mathrm{MHz}$ with a bandwidth of $100 \mathrm{MHz}$. The red colored plots indicate the AMbased synchronization retrieval method and the blue plots the FM-based synchronization retrieval method. All plots are averaged in time. (a)(b) Results of $T_{\text {image }}$ at $3.472 \mathrm{kSps}$ and $f_{\text {image. }}$ (c)(d) Results of $T_{\text {line }}$ at $10 \mathrm{MSps}$ and $f_{\text {line }}$.

\section{F. Video Image Leakage Detection, Synchronization Recovery and Reconstruction}

The methods of detecting a video image leak, recovering the video synchronization parameters and finally reconstructing the video image are only briefly described in the following section. The more elaborated description of these methods can be found back in our previous work [8], [28].

An autocorrelation method is applied, after the demodulation step, to recover the video synchronization information. This is done for both amplitude and frequency demodulated signals. The video synchronization information consists out of three time periods, the time period of one pixel, of one video line and of one video image frame [29]. By using autocorrelation methods, the end of each video line can be accurately extracted from the video leakage channels which is translated into the line frequency $f_{\text {line }}$. By selecting an arbitrary realistic number of pixels in the horizontal x-direction $n_{x}$ such as 2000 , and by extracting the line frequency $f_{\text {line }}$ and the image frequency $f_{\text {image }}$ from the synchronization recovery method, the pixel frequency and the number of pixels in the vertical y-direction $n_{y}$ can be determined.

Finally, the demodulated signal is resampled according to the determined pixel frequency and then displayed with a correct determined image resolution. A moving average is applied to these images to increase the readability of the reconstructed video images. The moving average length corresponds to the consecutive number of images that is averaged. Another technique which is used applies a single-pole infinite impulse response (IIR) filter to each image. Its response is

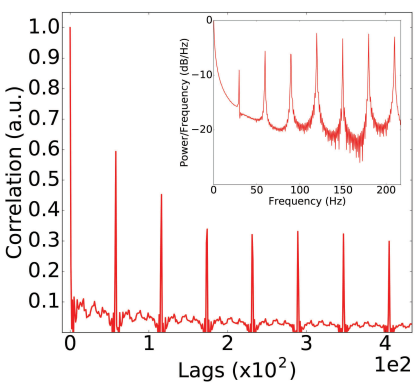

(a)

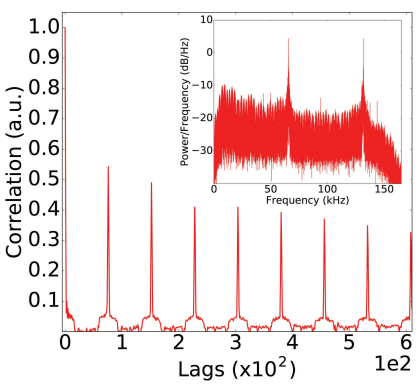

(c)

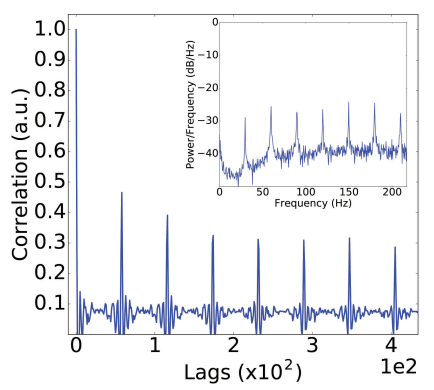

(b)

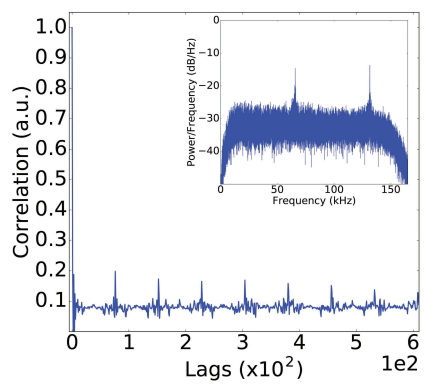

(d)
Fig. 9: Synchronization plots of test setup II.A at a $f_{\text {center }}$ of $525 \mathrm{MHz}$ tested with a bandwidth of $50 \mathrm{MHz}$. The red colored plots indicate the AM based synchronization retrieval method and the blue plots the FM based synchronization retrieval method. All plots are averaged in time. (a)(b) Results of $T_{\text {image }}$ at $1.736 \mathrm{kSps}$ and $f_{\text {image. }}$ (c)(d) Results of $T_{\text {line }}$ at $5 \mathrm{MSps}$ and $f_{\text {line }}$.

controlled by the decay value $\alpha$. The advantage is that the size of the memory buffer always equals one image. However, the disadvantages are that it is more difficult to exactly specify the images that need averaging and it is difficult to apply on non-static video images.

\section{G. The Tested VDU Setups}

A video image reconstruction method based on amplitude and frequency demodulation is proposed and is tested on several VDU setups. Most of today's VDUs employ HDMI cables for high definition and UHD images. Therefore, this signaling cable in combination with an UHD display is tested to determine their leakage response. The tests are done in a typical office environment that is located in an urban area. Hence, the local frequency spectrum includes occupied telecommunication channels, TV broadcasting signals, and sporadic emissions of electronic equipment in the nearvicinity.

The VDU displays a test image as depicted in Fig. 4b. The test image contains graphics representing horizontal, vertical, diagonal and circular lines. Glyphs are included such as the Roman and Greek alphabet in different font sizes and colors and with different color backgrounds. The purpose of this test image is to have a good frame of reference of all possible video graphics. The VDU is placed at a distance of 10 meters from the antenna system to analyze the performance of both reconstruction methods. This is a good representative distance for a possible eavesdrop attack. Although larger test distances are feasible, these are not tested due to fact that it would shift 


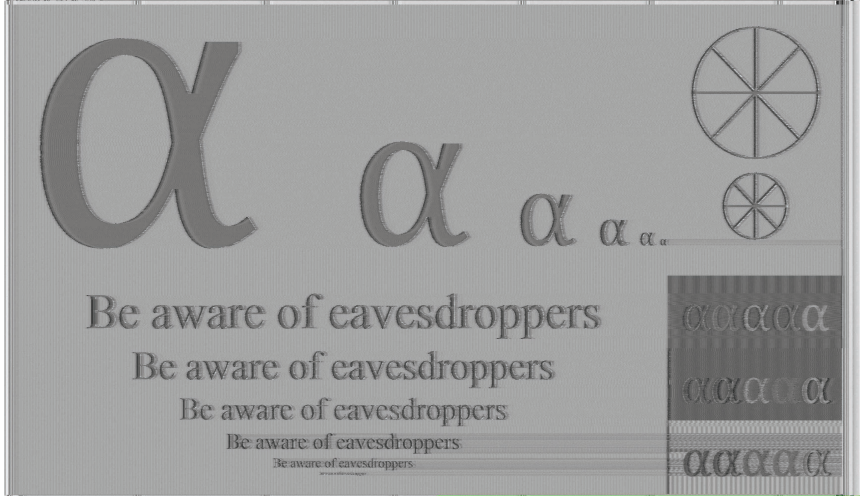

(a)

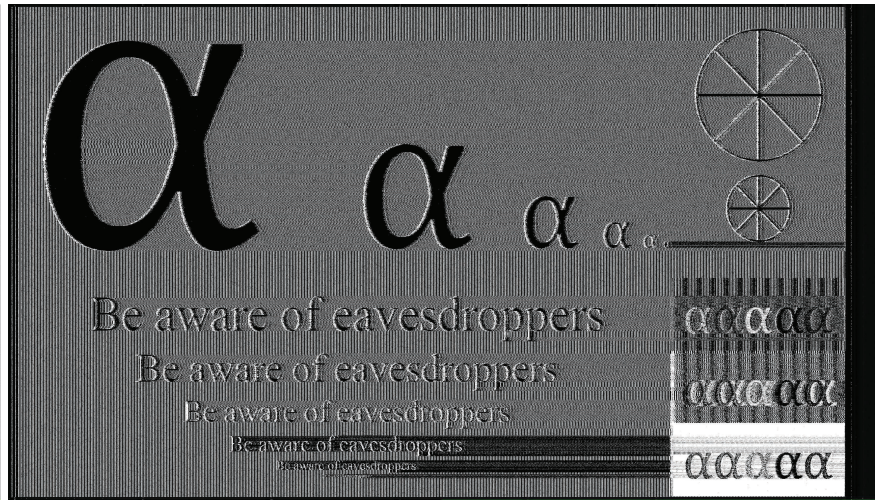

(b)

Fig. 10: Reconstructed video images of test setup I.A at the $f_{\text {center }}$ of $580 \mathrm{MHz}$ with a bandwidth of $100 \mathrm{MHz}$. The determined pixel frequency equals $52201843.150 \mathrm{~Hz}$. (a) AM based reconstruction method. The video image is averaged 50 times. (b) FM based reconstruction method. The video image is averaged 50 times.

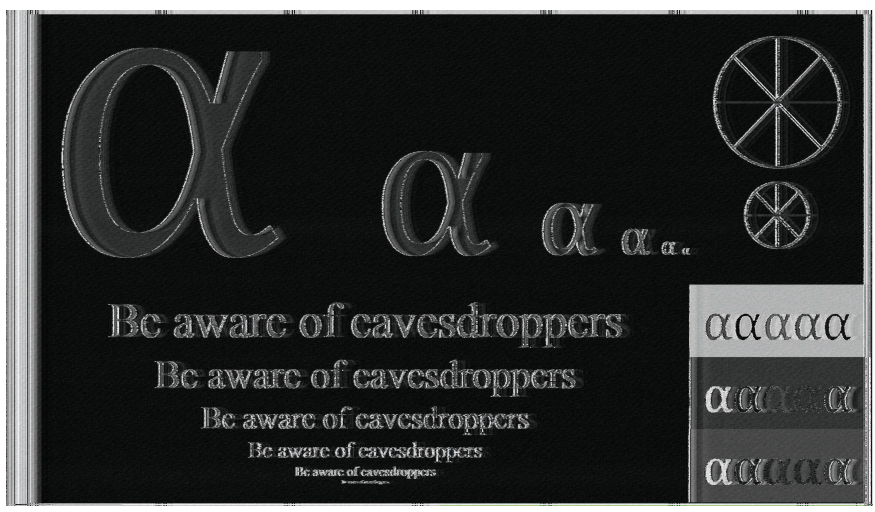

(a)

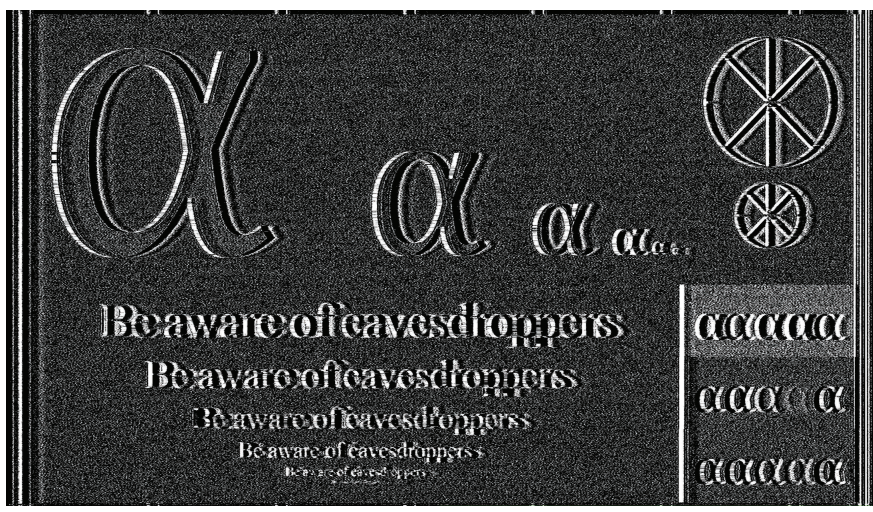

(b)

Fig. 11: Reconstructed images of test setup II.A at the $f_{\text {center }}$ of $525 \mathrm{MHz}$ and with a bandwidth of $50 \mathrm{MHz}$. The determined pixel frequency equals $65753612.562 \mathrm{~Hz}$ (a) AM based reconstruction method. The video image is averaged 50 times. (b) FM reconstruction method. The video image is averaged 100 times.

the focus of this paper too much towards an analysis of the SNR of the detected leakage signal and not to the comparison and the evaluation of the video image reconstruction methods.

The tested video setups are stated in Table I. All the test setups use an UHD LCD display with an image resolution of 3840x2160. The HDMI cables are manufactured by different companies and are a standard type of HMDI cables found in every office and household. Different HDMI cables are tested to verify if the other HDMI cables also suffer from the leakage phenomenon. For most of the tests, notebook II is used due to the lower leakage signal strength compared to the other notebook which makes for a better assessment of the effectiveness of the employed video reconstruction methods. The results of these test setups reveal whether the HDMI cable acts as a considerable video leakage source, hence these results also assess the proposed video leakage concepts of differential signaling cables.

\section{Results OF AM-BASED AND FM-BASED VideO IMAGE RECONSTRUCTION METHODS}

\section{A. Results of test setups I.A and II.A}

Firstly, leaking video channels are detected for both setups I.A and II.A when doing a frequency spectrum sweep from
410 to $790 \mathrm{MHz}$. The frequency spectra for both setups are displayed in Fig. 7. The detected video image leakage channels are indicated by a blue cross. The measurement system detected three video leakage channels with a relative high SNR at $537 \mathrm{MHz}, 598 \mathrm{MHz}$ and $626 \mathrm{MHz}$ for the setup I.A compared to two video leakage channels at $525 \mathrm{MHz}$ and 715 $\mathrm{MHz}$ for the setup II.A. The frequency spectrum also reveals important information about the frequency bands that are occupied. When a video channel is captured, these occupied frequency bands need to be considered when selecting the bandwidth of the receiver. Otherwise, too much noise of these occupied bands is taken in.

By applying the synchronization retrieval methods on a specific video leakage channel, one obtains the $f_{\text {line }}$ and $f_{\text {image }}$ of the leaking VDU. The results of the synchronization retrieval methods based on AM and FM are depicted in Fig. 8 for the setup I.A and in Fig. 9 for the setup II.A. The line and image synchronization information is depicted as PSD and autocorrelation plots. The plots reveal that the notebooks operate at different synchronization frequencies which in turn explains the difference in leakage spectrum. When analyzing and comparing the synchronization retrieval plots of the AM and FM based methods, the AM based method generally 


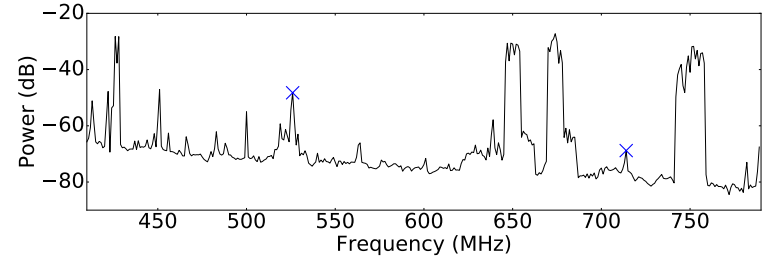

(a)

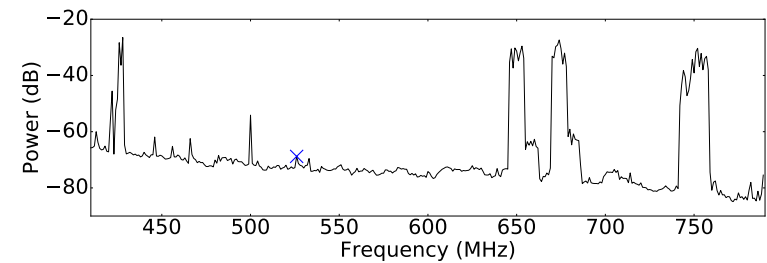

(b)

Fig. 12: Frequency spectra of test setups $I I . B$ and $I I . C$ from $410 \mathrm{MHz}$ to $790 \mathrm{MHz}$ with a resolution of $1 \mathrm{MHz}$ including the detected leakage channels indicated by a blue cross. Both spectra are averaged in time. (a) Test setup II.B. (b) Test setup II.C.

performs better for detecting the line frequency in terms of signal strength and the FM based method is more suited for image frequency detection in terms of a higher DC noise rejection. In terms of accuracy, the methods are very dependent on the nature of the leaked signal itself. Overall, both methods perform well in detecting the correct synchronization. Consequently, by combining the two methods, the performance of the synchronization retrieval method is improved.

After determining the synchronization frequencies, the data stream is resampled according the recovered $f_{\text {pixel }}$ and displayed according to the corresponding video image resolution. The results of the test setup I.A are displayed in Fig. 10.

Due to the fact that the test setup I.A emits multiple leakage channels in a wide frequency band and that the band from 500 $\mathrm{MHz}$ to $640 \mathrm{MHz}$ is relatively unoccupied, a large bandwidth of $100 \mathrm{MHz}$ at the center frequency of $580 \mathrm{MHz}$ could be opted for without taking into much noise. Because the UHD video display operates at a higher pixel frequency of $250 \mathrm{MHz}$ to $500 \mathrm{MHz}$, information is lost when the receiver's bandwidth is set to a lower frequency. Usually the receiver's bandwidth is set to $50 \mathrm{MHz}$ or lower to minimize band overlap with occupied bands. In this specific case, a $100 \mathrm{MHz}$ wide signal could be captured, reducing the error in signal bandwidth matching such that the AM based video reconstruction results in a very clear image. Only the smallest font size of 5 points is not fully reconstructed. The colored backgrounds in the bottom right corner show a specific pattern of vertical stripes depending on the color. These patterns relate to the used bit words of the original video data signal as revealed in section II-B.

The FM-based reconstruction acts a bit more like an edge detector compared to the AM based method. Another observation of the FM based video image reconstruction is the different reconstructed font colors of the small $\alpha^{\prime} s$ at the bottom left corner. These differences give the eavesdropper extra video image information of the target VDU and can be exploited.
Next, the video leakage channels of the test setup II.A are investigated. This time, a lower receiver bandwidth of $50 \mathrm{MHz}$ is chosen at a center frequency of $525 \mathrm{MHz}$. The reconstructed images of both methods result in a good readability and a high quality as seen in Fig. 11. Again, the main differences between the reconstructions are that the FM method acts more like an edge detection of glyphs and graphics, as discussed in the previous section. Also, the left edges are more highlighted than the right edges of the graphics. This is clearly seen in Fig. 11b. This can be explained by considering the rise and fall time of the differential signal transmitted through the HDMI cable. When one of the differential signal drivers generates a faster rise time than the other complementary signal driver or vice versa, a common-mode signal is created, ultimately resulting in a different frequency modulated leakage pattern. This was elaborated further in previous section II-A.

An interesting observation is that the small black colored letter $\alpha$ on a blue background seen in the test video image (Fig. 4b) is barely reconstructed for the AM-based reconstruction methods but is clearly visible in the FM-based reconstruction. This phenomenon has already been addressed in literature as the so-called TEMPEST fonts [4], [30] which are a collection of fore- and background color combinations which reduce the video leakage emissions. Hence, the same effect is observed here however by combining both reconstruction methods, this countermeasure becomes less effective.

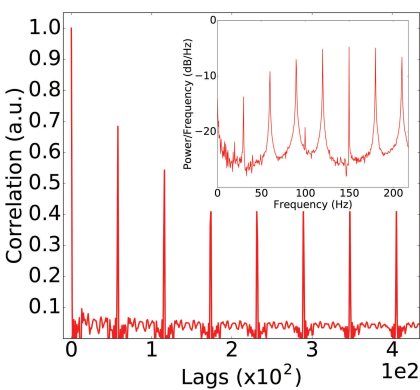

(a)

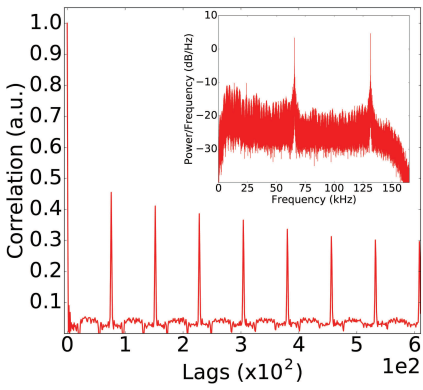

(c)

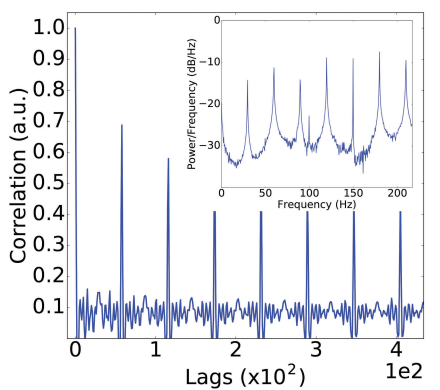

(b)

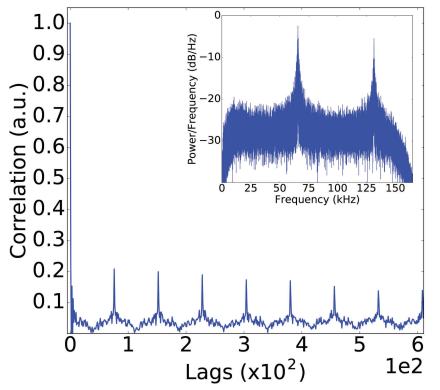

(d)
Fig. 13: Synchronization plots for test setup $I I . B$ at the $f_{\text {center }}$ of 525 $\mathrm{MHz}$ with a bandwidth of $50 \mathrm{MHz}$. The red colored plots indicate the AM based synchronization retrieval method and the blue plots the FM based synchronization retrieval method. All plots are averaged in time. (a)(b) Results of $T_{\text {image }}$ at $1.736 \mathrm{kSps}$ and $f_{\text {image }}$ (c)(d) Results of $T_{\text {line }}$ at $5 \mathrm{MSps}$ and $f_{\text {image. }}$ 


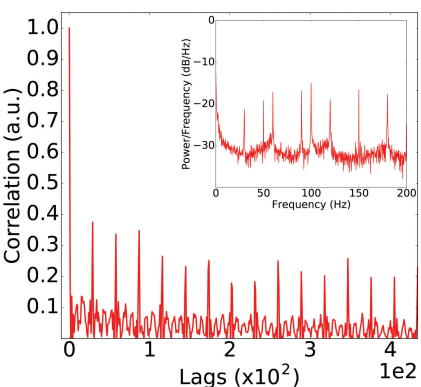

(a)

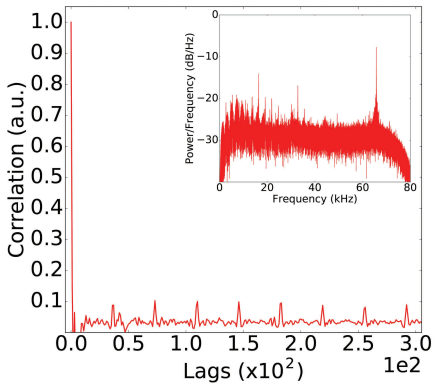

(c)

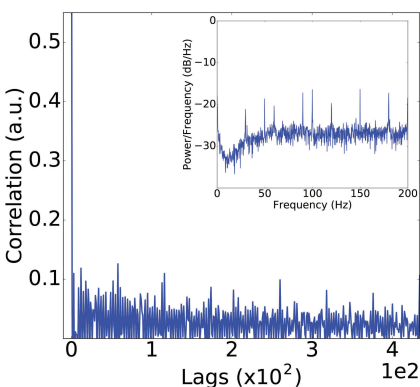

(b)

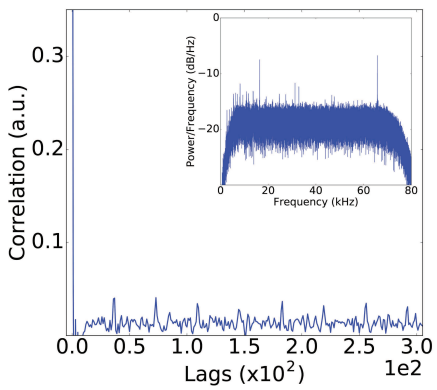

(d)
Fig. 14: Synchronization plots for test setup II.C at the $f_{\text {center }}$ of 525 $\mathrm{MHz}$ with a bandwidth of $25 \mathrm{MHz}$. The red colored plots indicated the AM based synchronization retrieval method and the blue plots the FM based synchronization retrieval method. All plots are averaged in time. (a)(b) Results of $T_{\text {image }}$ at $868 \mathrm{Sps}$ and $f_{\text {image. }}$ (c)(d) Results of $T_{\text {line }}$ at $2.5 \mathrm{MSps}$ and fine.

\section{B. Results of Test setups II.B and II.C}

First, the frequency spectra from $410 \mathrm{MHz}$ to $790 \mathrm{MHz}$ are captured for both setups. These are plotted in Fig. 12. For the setup with HDMI B, frequency peaks at $525 \mathrm{MHz}$ and 715 $\mathrm{MHz}$ are detected containing video leakage emanations. The setup with HDMI C only shows one leakage channel at 525 $\mathrm{MHz}$. The synchronization retrieval method is again applied for each setup and the results are depicted in Fig. 13 and Fig. 14.

As expected, the video leakage spectra and synchronization information is more or less the same as for the test setup $I I . A$ with only some pixel frequency offset of maximum $100 \mathrm{~Hz}$, which is due to the temperature-dependent oscillating quartz crystal inside the clock generators of the VDU [8]. The reconstructed video images of both setups are displayed in Fig. 15.

A general analysis of the reconstructed images reveals that even though the leaking emanations of test setup II.C are significantly lower, by having a longer acquisition and averaging time, the reconstructed image shows more or less the same readability as the other reconstructed images. It should be noted that the FM-based reconstruction method didn't perform as well for test setup II.C compared to the AM-based method as it contained much more noise. When test setup II.C is further analyzed, one observes that four $\alpha$ letters on the colored background for the AM-based reconstruction are not visible. However three of them are visible on the FMbased reconstructed image.

By examining the result of all video test setups, one can conclude that the HDMI cable is a major source of video leakage emanations. This conclusion is drawn from the fact that the only differences between the test setups of case II are the utilized HDMI cables. However each test setup has an unique video leakage response and consequently an unique video image reconstruction.

\section{Conclusion}

This research work reveals that modern video display equipment which use differential signaling cables such as high speed HDMI cables still suffer greatly from compromising video emanations. Exploiting the video leakage channels, the video images can be reconstructed to a high level of detail using reconstruction methods based on frequency or amplitude demodulation. Furthermore, a concept which explains the video leakage principles of differential signaling cables is proposed. The leakage emanations of an LVDS cable are closely examined by capturing its near- and far-field emissions and the differential signal on the LVDS cable simultaneously. It is confirmed that a significant common-mode noise signal is generated on the LVDS signal lines which results in leakage emissions that are correlated to the video image signal. Consequently, we conclude that video data signaling technologies that employ differential signaling pose a serious risk for video data security due to potential video leakages at large distances.

Several video test setups that utilize different HDMI cables are tested to analyze their video leakage response. These video setups also include an UHD video display and two notebooks which are tested at a distance of 10 meters. In addition, a novel video image leakage reconstruction method is proposed which detects and exploits compromising emanations originating from a VDU using frequency demodulation techniques. The method is proven to be effective for detecting and reconstructing leaking video emanations. The results of the

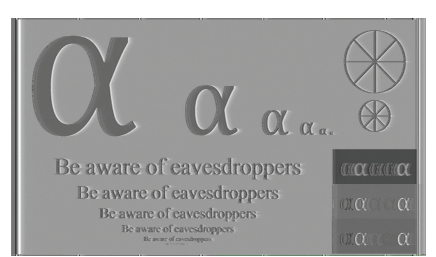

(a)

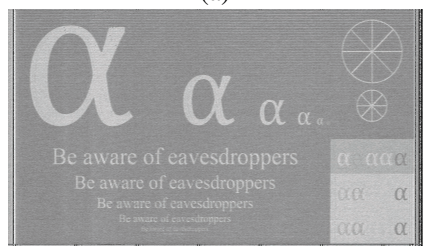

(c)

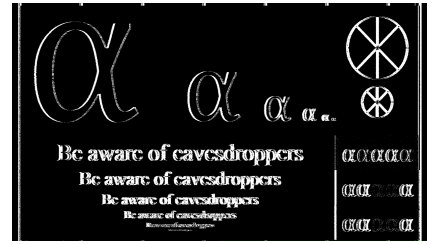

(b)

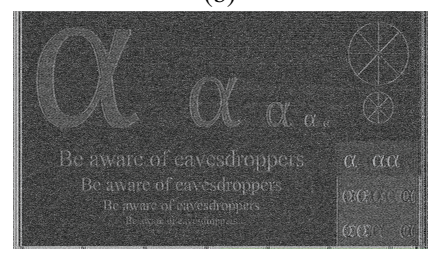

(d)
Fig. 15: Reconstructed images of test setups $I I . B$ and $I I . C$ at the $f_{\text {center }}$ of $525 \mathrm{MHz}$. (a) Test setup II.B. AM-based reconstruction method with a bandwidth of $50 \mathrm{MHz}$. The video image is averaged 100 times. (b) Test setup II.B. FM-based reconstruction method with a bandwidth of $50 \mathrm{MHz}$. The video image is averaged 100 times. (c) Test setup II.C. AM-based reconstruction method with a bandwidth of $25 \mathrm{MHz}$. The video image is averaged 800 times. (d) Test setup II.C. FM-based reconstruction method with a bandwidth of $25 \mathrm{MHz}$. The video image is averaged by setting the IIR decay parameter $\alpha$ to 0.005 . 
test setups are also compared to a video image reconstruction method based on amplitude demodulation. One can conclude that both video reconstruction methods have their unique response to the video leakage emanations, thus also having their own strengths. Hence combining both methods increases the success of obtaining video image information.

\section{REFERENCES}

[1] R. Frankland and A. Offences, "Side channels, compromising emanations and surveillance: Current and future technologies," Department of Mathematics, Royal Holloway, University of London, Egham, Surrey TW20 OEX, England, Tech. Rep. RHUL-MA-2011-07, 2011.

[2] S. Pennesi and S. Sebastiani, "Information security and emissions control," in 2005 International Symposium on Electromagnetic Compatibility, 2005. EMC 2005., vol. 3. IEEE, 2005, pp. 777-781.

[3] W. van Eck, "Electromagnetic Radiation from Video Display Units: An Eavesdropping Risk?" Comput. Secur, vol. 4, no. 4, pp. 269-286, dec 1985. [Online]. Available: http://dx.doi.org/10.1016/01674048(85) $90046-X$

[4] M. G. Kuhn, "Compromising emanations: eavesdropping risks of computer displays," Comput. Lab, Univ. Cambridge, Cambridge, U.K., Tech. Rep. 577, 2003

[5] H. Tanaka, O. Takizawa, and A. Yamamura, "A trial of the interception of display image using emanation of electromagnetic wave," Journal of the National Institute of Information and Communications Technology, vol. 52, no. 1-2, pp. 213-223, 2005.

[6] Y. Hayashi, N. Homma, Y. Toriumi, K. Takaya, and T. Aoki, "Remote visualization of screen images using a pseudo-antenna that blends into the mobile environment," IEEE Transactions on Electromagnetic Compatibility, vol. 59, no. 1, pp. 24-33, Feb 2017.

[7] H. S. Lee, D. H. Choi, K. Sim, and J. Yook, "Information Recovery Using Electromagnetic Emanations From Display Devices Under Realistic Environment," IEEE Transactions on Electromagnetic Compatibility, pp. $1-9,2018$.

[8] P. De Meulemeester, B. Scheers, and G. A. E. Vandenbosch, "A quantitative approach to eavesdrop video display systems exploiting multiple electromagnetic leakage channels," IEEE Transactions on Electromagnetic Compatibility, pp. 1-10, 2019.

[9] National Security Agency, "NACSIM 5000 TEMPEST FUNDAMENTALS," Fort Meade, MD, USA, Tech. Rep., 1982.

[10] H. Sekiguchi and S. Seto, "Proposal of an information signal measurement method in display image contained in electromagnetic noise emanated from a personal computer," Conference Record - IEEE Instrumentation and Measurement Technology Conference, pp. 1859-1863, 2008.

[11] M. G. Kuhn, "Compromising emanations of LCD TV sets," IEEE Transactions on Electromagnetic Compatibility, vol. 55, no. 3, pp. 564 570,2013

[12] Y. Hayashi, N. Homma, M. Miura, T. Aoki, and H. Sone, "A threat for tablet pcs in public space: Remote visualization of screen images using em emanation," in Proceedings of the 2014 ACM SIGSAC Conference on Computer and Communications Security. ACM, 2014, pp. 954-965.

[13] T. Song, Y. Jeong, and J. Yook, "Modeling of Leaked Digital Video Signal and Information Recovery Rate as a Function of SNR," IEEE Transactions on Electromagnetic Compatibility, vol. 57, no. 2, pp. 164 172, apr 2015.

[14] N. Zhang, Y. Lu, Q. Cui, and Y. Wang, "Investigation of Unintentional Video Emanations from a VGA Connector in the Desktop Computers," IEEE Transactions on Electromagnetic Compatibility, vol. 59, no. 6, pp. 1826-1834, 2017.

[15] A. Sayakkara, N.-A. Le-Khac, and M. Scanlon, "Accuracy enhancement of electromagnetic side-channel attacks on computer monitors," in Proceedings of the 13th International Conference on Availability, Reliability and Security. ACM, 2018, p. 15.

[16] B. Archambeault, J. C. Diepenbrock, and S. Connor, "Emi emissions from mismatches in high speed differential signal traces and cables," in 2007 IEEE International Symposium on Electromagnetic Compatibility, July 2007, pp. 1-6.

[17] T. Song, Y. Jeong, and J. Yook, "Modeling of leaked digital video signal and information recovery rate as a function of snr," IEEE Transactions on Electromagnetic Compatibility, vol. 57, no. 2, pp. 164-172, April 2015.

[18] "How to Map RGB Signals to LVDS/OpenLDI(OLDI) Displays (SNLA014A)," Texas Instruments Incorporated, Tech. Rep., 2018.
[19] P. De Meulemeester, B. Scheers, and G. A. Vandenbosch, "Reconstructing Video Images in Color Exploiting Compromising Video Emanations ("forthcoming")," 20202019 International Symposium on Electromagnetic Compatibility - EMC EUROPE, p. 6, 2020.

[20] Y. Suzuki and Y. Akiyama, "Jamming technique to prevent information leakage caused by unintentional emissions of PC video signals," in IEEE International Symposium on Electromagnetic Compatibility, vol. 768, 2010, pp. 132-137.

[21] Y.-i. Hayashi, "State-of-the-art research on electromagnetic information security," Radio Science, vol. 51, no. 7, pp. 1213-1219, 2016.

[22] H. Sekiguchi, "Measurement of radiated computer RGB signals," Progress In Electromagnetics Research, vol. 7, pp. 1-12, 2009.

[23] M. Vuagnoux and S. Pasini, "An improved technique to discover compromising electromagnetic emanations," in 2010 IEEE International Symposium on Electromagnetic Compatibility. IEEE, 2010, pp. 121126.

[24] B. B. Yilmaz, A. Zaji, and M. Prvulovic, "Modelling jitter in wireless channel created by processor-memory activity," in 2018 IEEE International Conference on Acoustics, Speech and Signal Processing (ICASSP), April 2018, pp. 2037-2041.

[25] M. Prvulovic, A. Zajic, R. L. Callan, and C. J. Wang, "A Method for Finding Frequency-Modulated and Amplitude-Modulated Electromagnetic Emanations in Computer Systems," IEEE Transactions on Electromagnetic Compatibility, vol. 59, no. 1, pp. 34-42, feb 2017.

[26] P. De Meulemeester, L. Bontemps, B. Scheers, and G. A. E. Vandenbosch, "Synchronization retrieval and image reconstruction of a video display unit exploiting its compromising emanations," in 2018 International Conference on Military Communications and Information Systems (ICMCIS), May 2018, pp. 1-7.

[27] D. E. Foster and S. W. Seeley, "Automatic Tuning, Simplified Circuits, and Design Practice," Proceedings of the Institute of Radio Engineers, vol. 25, no. 3, pp. 289-313, mar 1937.

[28] P. De Meulemeester, B. Scheers, and G. A. Vandenbosch, "Eavesdropping a (Ultra-)High-Definition Video Display from an 80 Meter Distance Under Realistic Circumstances ("forthcoming")," 2020 IEEE International Symposium on Electromagnetic Compatibility, Signal Integrity and Power Integrity, p. 6, 2020.

[29] Video Electronics Standards Association (VESA), "VESA and Industry Standards and Guidelines for Computer Display Monitor Timing (DMT) Version 1, Revision 11," Milpitas, CA, USA, Tech. Rep., 2007.

[30] M. G. Kuhn and R. J. Anderson, "Soft tempest: Hidden data transmission using electromagnetic emanations," in International Workshop on Information Hiding. Springer, 1998, pp. 124-142. 